Urología N eurológica y Urodinámica

Arch. Esp. Urol., 58, 2 (131-138), 2005

\title{
PREVALENCIA DE VEIGA HIPERACTIVA EN ESPAÑA: ESTUDIO POBLACIONAL
}

\author{
David Castro ${ }^{1}$, M ontserrat Espuña ${ }^{2}$, M arta Prieto ${ }^{3}$ y Xavier Badia ${ }^{4}$.
}

Servicio de Urología ${ }^{1}$, Hospital Universitario de Canarias, Sta. Cruz de Tenerife.

Institut C línic de G inecología ${ }^{2}$, O bstetricia i N eonatologia Hospital C línic, Universitat de Barcelona. Barcelona.Departamento M édico3. Yamanouchi Pharma, S.A. Madrid.

Health 0 utcomes Research Europe ${ }^{4}$. Barcelona. España.

\begin{abstract}
Resumen.- O BJETIVO : Conocer la prevalencia de la sintomatología compatible con vejiga hiperactiva $(\mathrm{VH})$ según la definición de la International Continence Society (IC S) 2002 que incluye pacientes con urgencia miccional, con o sin incontinencia urinaria de urgencia generalmente acompañada de frecuencia y nicturia. MÉTO DO : Se realizaron 1669 entrevistas telefónicas efectivas a hombres y mujeres $\geq 40$ años. Para el cálculo del tamaño muestral se partió de la prevalencia estimada de VH estratificada por edad y género extraída del artículo de Milsom y col al observarse una gran variabilidad en los diferentes estratos. Se recogieron datos sobre aparición y prevalencia de los principales síntomas de vejiga hiperactiva así como diagnósticos médicos y tratamiento por condiciones urinarias.
\end{abstract}

David Castro.

Servicio de Urología.

Hospital Universitario de Canarias

$\mathrm{C} / \mathrm{O}$ fra $\mathrm{s} / \mathrm{n}$

38320 San C ristobal de la Laguna.

Sta. Cruz de Tenerife. (España)
RESULTADOS: La muestra poblacional consistió en 1669 sujetos $\geq 40$ años con un porcentaje de mujeres del $50,6 \%$ y de varones del $49,4 \%$. La prevalencia de la sintomatología compatible con VH según la definición de la ICS fue del $21,5 \%$, siendo significativamente mayor en las mujeres $(25,6 \%)$ que en los hombres $(17,4 \%)(p<0,05)$. Ajustando estos resultados a la población española según el censo del año 2000 del INE, dicha prevalencia se situó en el $19.9 \%$, siendo igualmente superior en mujeres (23.6\%) que en hombres (15.4\%). La incontinencia urinaria (IU) de urgencia y la IU de esfuerzo también fueron significativamente superiores en mujeres que en varones $(16,7 \%$ vs $10,4 \%$ y $33,1 \%$ vs $7,9 \%$ respectivamente) ( $p<0,01)$. El $9,8 \%$ de las mujeres y el $7,9 \%$ de los varones encuestados referían una frecuencia miccional $>8$ micciones/ día. El $62 \%$ de los varones y el $52,4 \%$ de las mujeres afirmaban levantarse por la noche para orinar. Un 52,1\% de los pacientes con sintomatología compatible con $\mathrm{VH}$ refirió haber visitado al médico por causas relacionadas con problemas urinarios mientras que, un 16,7\% declaró estar recibiendo tra tamiento por algún síntoma urinario.

CON CLUSIONES: La prevalencia de sintomatología compatible con VH obtenida en el presente estudio es alta, consistente con los datos recogidos en estudios internacionales. La urgencia miccional, síntoma que define la patología, es más prevalente en las mujeres españolas que en los hombres. Es necesaria la realización de estudios adicionales que puedan mejorar el conocimiento del impacto de la $\mathrm{VH}$ en la población general en España. 
Palabras clave: Vejiga hiperactiva. Prevalencia. Urgencia miccional. Incontinencia de urgencia. Frecuencia miccional.

Summary.- O BJECTIVES: The aim of this study was to assess the prevalence of the urinary symptoms suggestive of overactive bladder (OAB) in Spain based on the International Continence Society (ICS) 2002 consensus criteria as urinary urgency, with or without urge incontinence, usually with frequency and nocturia. METHODS: 1,669 real telephone interviews were conducted to adults aged $\geq 40$ years. The sample size estimation was made according to the prevalence for $O A B$ described in the Milsom paper stratified by age and gender due to the high variability observed between ranges. Appearance and prevalence of main $O A B$ symptoms, medical diagnostic and therapy $d$ ue to these symptoms data were collected.

RESULTS: the sample population was 1669 aged $\geq 40$ years, $50,6 \%$ women and $49,4 \%$ men. The

overall prevalence of symptoms suggestive of $O A B$ according to the $O A B$ definition from IC S report 2002 was $21,5 \%$, significantly higher in women $(25,6 \%)$ than men $(17,4 \%)(p<0,05)$. Adjusting these data to Spanish $N$ ational Census of year 2000 , the prevalence was $19.9 \%$, being higher as well in women $(23.6 \%)$ than men (15.4\%). Urge urinary incontinence and stress urinary incontinence were superior in women $(16,7 \%$ vs $10,4 \%$ and $33,1 \%$ vs $7,9 \%$ respectively) $(p<0,01)$. U rinary frequency $>8$ voids/ day was referred by $9,8 \%$ of women and $7,9 \%$ of men interviewed. $62 \%$ of men and $52,4 \%$ of women reported they get up at night to void. A total of $52,1 \%$ of patients with urinary symptoms suggestive of overactive bladder reported they had consulted a doctor anytime before the interview due to urinary disorders and 16,7\% w as currently receiving treatment for some of these symptoms.

CONCLUSION S: Prevalence of urinary symptoms suggestive of $O$ veractive Bladder is high in this study, in accordance with data from international studies. Urinary urgency, symptom which defines the pathology, is more prevalent in Spanish women than men. Further studies are needed to better assess OAB impact in the Spanish general population.

Keywords: 0 veractive bladder. Prevalence. Urinary urgency. Urge incontinence. Urinary frequency.

\section{INTRODUCCIÓN}

El síndrome de Vejiga Hiperactiva (VH) es una entidad clínica de marcada importancia entre adultos de edad avanzada (1). Son escasos los datos de prevalencia de esta patología en España. La prevalencia de $\mathrm{VH}$ en la población general española $\geq 40$ años de edad se estima del $22 \%$, según el estudio realizado por Milsom y col (2).

Clásicamente se consideraba que la $\mathrm{VH}$ se podía diagnosticar siempre que el paciente presentara los síntomas de urgencia miccional y/ o frecuencia miccional y/ o incontinencia de urgencia, y que dichos síntomas no pudieran explicarse por otra patología 0 condición metabólica (3). En el año 2002, la International Continence Society (ICS) modificó la definición según la cual se diagnostica la $\mathrm{VH}$ ante la presencia de urgencia miccional, con o sin incontinencia de urgencia, pudiendo a menudo asociarse a frecuencia miccional y nicturia (1).

De este modo los síntomas más característicos de VH son la urgencia miccional, la incontinencia de urgencia, y la frecuencia miccional, consecuencia de la presencia involuntaria de contracciones en la vejiga durante el proceso de llenado (1, 3-5). La presencia de estos mismos síntomas en otras patologías relacionadas con la próstata 0 en infecciones de orina, obligan a la realización de un buen diagnóstico diferencial $(6,7)$.

El diagnóstico de VH es cada vez más frecuente en las consultas de atención primaria, urología y ginecología, a pesar de lo cual se sospecha que la prevalencia observada de VH es inferior a la prevalencia real. Por un lado, un amplio porcentaje de personas es reticente a acudir a la consulta médica (8). Por otro lado, a pesar de haberse realizado diferentes estudios sobre esta patología, la utilización de definiciones diversas y las diferencias culturales de las distintas zonas geográficas donde se han realizado los trabajos, hacen difícil establecer unos márgenes exactos de prevalencia de $\mathrm{VH}$ (9-13).

La VH es una patología cuya aparición aumenta con la edad. El envejecimiento progresivo de la población española, junto al infradiagnóstico de esta enfermedad hacen necesario tener datos reales sobre el impacto de esta patología en España. El obje- 
tivo del presente estudio fue conocer la prevalencia actual de sintomatología compatible con VH según la nueva definición de la ICS.

\section{MATERIAL Y MÉTODOS}

Se realizó un estudio epidemiológico transversal en una muestra poblacional de 40 años 0 más, para detectar la presencia de sintomatología compatible con $\mathrm{VH}$, a través de una encuesta telefónica cerra$d a$, especialmente diseñada para el estudio. Las llamadas telefónicas se realizaron entre los meses de Marzo y Mayo de 2004, fueron asistidas por ordenador (CATI-Computer Assisted Telephone Interview) y tuvieron una duración media de 10 minutos. Previo a la realización de la entrevista los entrevistadores informaron a cada uno de los encuestados sobre la confidencialidad, el anonimato y el derecho a no responder a todas las preguntas, y se pidió así mismo su consentimiento para realizar dicha entrevista.

Para el cálculo del tamaño muestral se partió de la prevalencia estimada de $\mathrm{VH}$ estratificada por edad y género extraída del artículo de Milsom y col 2001 (2), al observar una gran variabilidad en los diferentes estratos. Este método permite minimizar el error muestral en cada estrato según edad y género recibiendo los estratos con mayor variabilidad un mayor tamaño muestral. Este muestreo se realizó según el método de asignación óptima por estrato de edad y género, aceptando un riesgo alfa de 0,05 y una precisión de \pm 5 unidades porcentuales, se estimó una muestra necesaria global de 1.669 personas.

Los individuos no localizados, fallecidos, desplazados 0 que no accedieron a responder a la entrevista, fueron sustituidos de forma aleatoria por otro sujeto elegible de la misma cuota.

Se desarrolló específicamente para este estudio una entrevista que revisó un grupo de expertos en uro-ginecología. La entrevista constaba de 23 preguntas estructuradas en dos partes. En la primera parte se preguntaban datos sociodemográficos como la edad, género, Comunidad Autónoma y hábitat de residencia, nivel de estudios, nivel social y situación laboral. En la segunda parte se preguntaba por la presencia 0 no de los diversos síntomas asociados a VH y de otros síntomas urinarios (Tabla I).

\section{A nálisis Estadístico}

Se realizó un análisis descriptivo de las características sociodemográficas y clínicas de la población de estudio. Para comparar la prevalencia según grupos de edad, género y región geográfica, así como la distribución de los distintos síntomas según grupo diagnóstico, se utilizó la prueba de la Chi-Cuadrado. Para inferir el dato de prevalencia global a la población general española, se ponderaron los resultados obtenidos en el estudio según las cifras del censo del 2000 del IN E estratificando por edad y género (14). El análisis de los datos se realizó utilizando el paquete estadístico SPSS 9.0 para W indows. En todas las pruebas estadísticas se consideró como nivel de significación $a=0,05$.

\section{RESULTADOS}

Se realizaron 4907 contactos telefónicos con el fin de alcanzar el tamaño muestral estimado.

La muestra poblacional efectiva consistió en 1669 sujetos $\geq 40$ años con un porcentaje de mujeres del $50,6 \%$ y de varones del $49,4 \%$. En la tabla II se describen las variables sociodemográficas de los sujetos entrevistados. El $50.6 \%$ de la muestra evaluada eran mujeres, el $67,3 \%$ eran mayores de 60 años y el $55,2 \%$ de los entrevistados eran jubilados o pensionistas.

En el 21,5\% (IC 95\% 19,5-23,5) de la población encuestada se registró la presencia de urgencia miccional con o sin incontinencia de urgencia, aspecto que definía la presencia de VH. La prevalencia de de sintomatología compatible con VH entre las mujeres fue del 25,6\%, significativamente superior a la referida entre los hombres $(17,4 \%)(p<0,05)$. Al igual que lo observado en otros estudios poblacionales la prevalencia global fue máxima en el grupo de mayor edad $(p<0,05)(2,15)$. A nalizado por género sólo en las mujeres aumentó la prevalencia de VH de forma progresiva y estadísticamente significativa con la edad $(p<0,05)$ (Tabla III). No hubo diferencias estadísticamente significativas en la prevalencia de $\mathrm{VH}$ según las diferentes regiones españolas. La prevalencia de VH ajustada a la población general española se situó en el 19.9\% (IC 95\% 18.0-21.8), siendo superior entre las mujeres $(23.6 \%)$ que en los hombres (15.4\%)

Evaluando los síntomas en el total de sujetos encuestados según grupos de género (Tabla IV), se 
- En la última semana, ¿cuántas veces al día ha ido a orinar?

1. Entre 1 y 4 veces

2. Entre 5 y 8 veces

3. Más de 8 veces

- ¿Cree que últimamente orina más veces de lo que era habitual en Ud.?

- Habitualmente, ¿se levanta por las noches a orinar?

- ¿Cuántas veces cada noche?

1. Entre 1 y 2 veces

2. Entre 3 y 4 veces

3. Más de 4 veces

- ¿ Desde cuándo le sucede?

- ¿Habitualmente, tiene de repente deseos irreprimibles de ir a orinar, ha ido corriendo al baño y ha llegado a tiempo?

- ¿Cuántas veces al día le sucede?

1. Entre 1 y 2 veces

2. Entre 3 y 4 veces

3. Más de 4 veces al día

- ¿ Desde cuándo le sucede?

- En la última semana, ¿alguna vez ha ido a orinar y no le ha dado tiempo de llegar al baño?

- ¿Cuántas veces le sucede?

1. 1 vez por semana

2. De 2 a 4 veces por semana

3. Cada día

- ¿Desde cuándo le pasa?

- Habitualmente, ¿se le escapa la orina al hacer ejercicio, toser, estornudar, levantarse, etc.?

- Habitualmente, ¿cuánto tiempo cree que pasa entre una vez que va a orinar y la siguiente?

- ¿Tiene molestias al orinar?

- Preguntar sólo en caso de HOMBRE ¿Tiene un chorro miccional débil y/ o interrumpido?

- ¿Tiene habitua Imente infecciones de orina?

- ¿Ha visitado alguna vez al médico por causas relacionadas con la micción (al orinar)?

- ¿Está recibiendo tratamiento para algún síntoma urinario? 
observa como la prevalencia de IU de urgencia fue menor en hombres que en mujeres $10,4 \%$ vs $16,7 \%$ $(p<0,01)$ aumentando con la edad en ambos géneros de forma estadísticamente significativa $(p<0,01)$. No hubo diferencias estadísticamente significativas en la proporción de mujeres y hombres que referían orinar más de 8 veces al día, 9,8 vs. 7,9\% respectivamente (Figura 1) y tampoco se detectaron cambios estadísticamente significativos con la edad. El 26,8\% de los sujetos encuestados refirió creer que últimamente orinaba más veces de lo que era habitual, porcentaje que ascendía al 48,7\% entre los sujetos que además presentaban urgencia miccional. Los varones referían levantarse por la noche a orinar en mayor medida que las mujeres 62 vs. $52,4 \%$ respectivamente $(p<0,01)$, aumentado de forma significativa con la edad en los dos grupos $(p<0,01)$ (Figura 1 ). Por el contrario la prevalencia de IU de esfuerzo, fue menor en hombres que en mujeres 7,9 vs. $33,1 \%,(p<0,01)$, aumentando también con la edad en ambos géneros de forma estadísticamente significativa $(p<0,01)$.

Se analizó la presencia de los distintos síntomas urinarios según la presencia o no de urgencia miccional, síntoma que define el síndrome de VH (1). Los pacientes con urgencia miccional refirieron en proporción significativamente superior $(p<0,05)$ una frecuencia miccional más de 8 veces al día (19\% vs. $6,2 \%)$, levantarse por la noche para orinar $(72,7 \%$ vs. $52,9 \%)$, IU de urgencia $(36,5 \%$ vs. $7,3 \%)$ e IU de esfuerzo (39\% vs. 15,60\%). O tros síntomas como molestias al orinar, infecciones de orina y, en hombres, chorro miccional débil y/ o interrumpido también fueron más frecuentes en pacientes con urgencia miccional $(7,8 \%, 13,9 \%, 51,7 \%$ vs. 3\%, 5,2\%,30,0\% respectivamente) $(p<0,01)$.

TABLA II. CARACTERISTICAS SO C IO DEM O GRÁFICAS DE LA PO BLACIÓ N TO TAL EN TREVISTADA.

\begin{tabular}{|c|c|c|c|c|}
\hline Características, n, (\%) & & Varones & Mujeres & Total \\
\hline \multirow{5}{*}{ GRUPOS DE EDAD } & $40-49$ & $68(8,3)$ & $132(15,6)$ & $200(12)$ \\
\hline & $50-59$ & $156(18,9)$ & $189(22,4)$ & $345(20,7)$ \\
\hline & $60-69$ & $258(31,3)$ & $220(26)$ & $478(28,6)$ \\
\hline & Más de 70 & $342(41,5)$ & $304(36)$ & $646(38,7)$ \\
\hline & Total & 824 & 845 & 1669 \\
\hline & & & & \\
\hline \multirow{6}{*}{ Nivel de Estudios } & Universitarios & $94(11,4)$ & $51(6)$ & $145(8,7)$ \\
\hline & Secundarios & $170(20,6)$ & $104(12,3)$ & $274(16,4)$ \\
\hline & Primarios & $395(47,9)$ & $460(54,4)$ & $855(51,2)$ \\
\hline & Sin Estudios & $135(16,4)$ & $187(22,1)$ & $322(19,3)$ \\
\hline & Analfabetos & $30(3,6)$ & $43(5,1)$ & $73(4,4)$ \\
\hline & Total & 824 & 845 & 1669 \\
\hline & & & & \\
\hline \multirow{4}{*}{ Nivel } & Alta & $12(1,5)$ & $11(1,3)$ & $23(1,4)$ \\
\hline & Media & $547(66,4)$ & $575(68)$ & $1122(67,2)$ \\
\hline & Baja & $265(32,2)$ & $259(30,7)$ & $524(31,4)$ \\
\hline & Total & 824 & 845 & \\
\hline \multirow{6}{*}{ Situación laboral } & Incapacidad Permanente & $8(1)$ & $8(0,9)$ & $16(1)$ \\
\hline & Jubilado/ pensionista & $576(69,9)$ & $346(40,9)$ & $922(55,2)$ \\
\hline & En Paro & $19(2,3)$ & $16(1,9)$ & $35(2,1)$ \\
\hline & Actualmente Trabaja & $219(26,6)$ & $134(15,9)$ & $353(21,2)$ \\
\hline & Ama de casa & $2(0,2)$ & $341(40,4)$ & $343(20,6)$ \\
\hline & Total & 824 & 845 & 1669 \\
\hline
\end{tabular}


Entre el total de sujetos entrevistados un $37,6 \%$ refirió haber visitado al médico por causas relacionadas con la micción, porcentaje que aumenta entre los pacientes con urgencia miccional $(52,1 \%)$. Un 9,6\% de los pacientes encuestados seguían algún tratamiento por síntomas urinarios en el momento de la entrevista, porcentaje que ascendió a 16,7\% entre los pacientes con urgencia miccional.

\section{DISCUSIÓN}

Se muestra en el presente estudio una prevalencia global de sintomatología compatible con VH de acuerdo a la definición consensuada por la ICS en 2002, que contempla la presencia de urgencia miccional con 0 sin incontinencia de urgencia, del 21,5\% (IC 95\% 19,5 - 23,5). Tras ajustar por la distribución de la población general española $\geq 40$ años, la prevalencia fue similar, 19,9\% (IC 95\% 18.0-21.8). Diversos estudios internacionales muestran resultados próximos a estos. Milsom y col. $(2,15)$ empleando la definición previa a la actual que incluía a los pacientes con síntomas de urgencia $y / 0$ frecuencia $y / 0$ incontinencia de urgencia, realizaron un estudio poblacional en varios países europeos, observando una prevalencia total de $16,6 \%$. En este último estudio la prevalencia varió entre un $12 \%$ en Francia e Italia, 18-19\% en A lemania, Inglaterra y Suecia, llegando a un máximo de $22 \%$ en España. Stewart y col. (15) llevaron a cabo un estudio de población general en EE.UU donde se observó una prevalencia global de $16,5 \%$; otro estudio realizado recientemente en Canadá muestra una prevalencia global de VH del 18,1\% (16).

Las diferencias en las poblaciones estudiadas, las definiciones utilizadas y los métodos para la obtención de datos, así como diferencias culturales de las poblaciones evaluadas que pueden en cierto modo condicionar la respuesta de los sujetos entrevistados, nos obligan a comparar estos estudios con cautela.

Los síntomas urinarios compatibles con VH fueron altamente prevalentes. Siguiendo la definición de consenso de la ICS 2002, la urgencia miccional es el síntoma que define la presencia del síndrome de VH. En el presente estudio, entre los pacientes con urgencia miccional, se observa que la incontinencia urinaria fue muy prevalente (IU de Urgencia 36,5\%; IU de esfuerzo $39 \%$ ). La prevalencia de ambos tipos de IU fue significativamente superior entre las mujeres. Estos datos coinciden con el estudio europeo de Milsom y col y el estudio realizado en los EE.UU, en los que se observó que la IU era más prevalente entre las mujeres $(2,15)$.

El $26,8 \%$ de los sujetos encuestados refirió creer que últimamente orinaba más veces de lo que era habitual mientras que únicamente el $8,9 \%$ consi-

TABLA III. DISTRIBUCIÓN DE SÍN TO MAS URIN ARIO S SEG ÚN EDAD Y GÉN ERO EN LA MUESTRA TO TALDE SUJETO S EN CUESTADOS.

\begin{tabular}{|c|c|c|c|}
\hline Mujeres & $\begin{array}{c}\text { Urgencia miccional } \\
216(25,6 \%)\end{array}$ & $\begin{array}{c}\text { IU de urgencia } \\
141(16,7 \%)\end{array}$ & $\begin{array}{c}>\mathbf{8} \text { micciones/ dia } \\
83(9,8 \%)\end{array}$ \\
\hline $40-49$ años & $20(15,2 \%)$ & $2(1,5 \%)$ & $13(9,8 \%)$ \\
\hline $50-59$ años & $41(21,7 \%)$ & $16(8,5 \%)$ & $17(9,0 \%)$ \\
\hline $60-69$ años & $54(24,5 \%)$ & $26(11,8 \%)$ & $24(10,9 \%)$ \\
\hline$>70$ años & $101(33,2 \%)$ & $97(31,9 \%)$ & $29(9,5 \%)$ \\
\hline Hombres & $143(17,4 \%)$ & $86(10,4 \%)$ & $65(7,9 \%)$ \\
\hline $40-49$ años & $7(10,3 \%)$ & & $1(1,5 \%)$ \\
\hline $50-59$ años & $29(18,6 \%)$ & $7(4,5 \%)$ & $9(5,8 \%)$ \\
\hline $60-69$ años & $37(14,3 \%)$ & $18(7,0 \%)$ & $21(8,1 \%)$ \\
\hline$>70$ años & $70(20,5 \%)$ & $61(17,8 \%)$ & $34(9,9 \%)$ \\
\hline
\end{tabular}


FIG URA 1. Prevalencia de IU de Esfuerzo y de pacientes que refieren levantarse por la noche para orinar según género y edad en el total de la muestra encuestada.
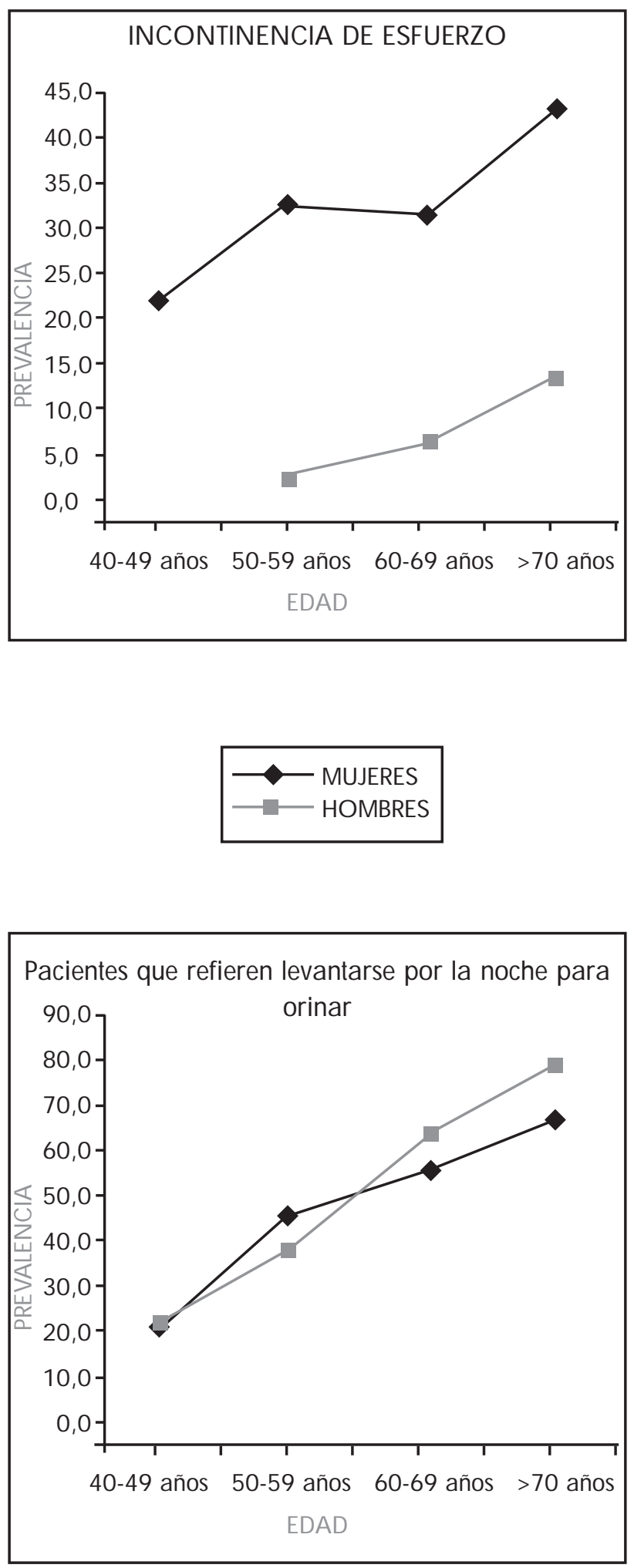

deraba que el número de micciones/ día era $>8$. En cualquier caso, estas preguntas no iban ligadas a la cumplimentación de un diario miccional, ni a una entrevista directa médico-paciente siendo éstas limitaciones en la medición de la frecuencia miccional en la que la memoria puede fallar al concretar un número exacto de micciones.

La prevalencia de pacientes que refirió levantarse por la noche para orinar fue alta $(57,2 \%$ del total de encuestados y $72 \%$ de los pacientes con urgencia miccional). Probablemente esta prevalencia sea tan alta dado el carácter de la pregunta (no se recoge si realmente se despertaban por el deseo de orinar) y por el alto porcentaje de sujetos mayores de 60 años incluidos en la encuesta.

El $52,1 \%$ de los pacientes con sintomatología compatible con VH había visitado al médico por alguna causa relacionada con la micción, dato similar al observado en el estudio de Milsom y col, en el que los pacientes con VH que habían acudido al médico asciende al $60 \%$. Únicamente el $16,7 \%$ de los pacientes con sintomatología urinaria compatible con $\mathrm{VH}$ estaba recibiendo algún tratamiento para sus síntomas miccionales, tasa muy parecida a la encontrada en el estudio de Milsom y col, y que evidencia la aún baja tasa de tratamiento de esta patología (2).

Hay que destacar el dato de que en los sujetos con urgencia miccional fue mas frecuente el antecedente de chorro débil y/ o interrumpido, molestias al orinar o infección de orina, sin disponer de información adicional para confirmar en estos casos la existencia de otra patología. Este es el primer estudio de ámbito nacional publicado en España sobre prevalencia de sintomatología compatible con VH utilizando la definición del la ICS 2002.

\section{CONCLUSIONES}

La prevalencia de sintomatología compatible con $\mathrm{VH}$ en este estudio es alta, consistente con los datos publicados en estudios internacionales. La urgencia miccional, síntoma que define la patología, es más prevalente en las mujeres españolas que en los hombres. Es necesaria la realización de estudios adicionales que puedan mejorar el conocimiento del impacto de la VH en la población general en España. 


\section{BIBUOGRAFIA y LECTURAS \\ RECOMENDADAS (*lectura de interés y $* *$ lectura fundamental)}

**1. ABRAMS, P.; CARDOZO, L.; FALL, M. y cols.: "The standardisation of terminology of lower urinary tract function: report from the Standardisation Sub-commitee of the International Continence Society". Neurology and Urodynamics, 21: 167, 2002.

**2. MILSOM, I.; ABRAMS, P.; CARDOZO, L. y cols.: "How widespread are the symptoms of an overactive bladder and how are they managed? A populationbased prevalence study". BJU International, 87: 760, 2001.

3. ABRAMS, P.; WEIN, A.J.: "The overactive bladder, a widespread but treatable condition". Stockholm: Sparre Medical Group, 1998.

*4. VAN BRUMMEN, P.; VAN DER VAART, H.; HEINTZ, P.: "How well can overactive bladder symptoms be objectively measured? [Abstract]". The International Continence Society Congress, Heildeberg, 2002.

5. ELLIOT, D.; LIGHTNER, D.; BLUTE, M.. "Medical management of overactive bladder". Mayo Clin. Proc., 76: 353, 2001.

6. "Incontinencia urinaria, un problema con soluciones". Sístole, 21, 2001.

7. AESGP Foundation: "Overactive bladder / urge incontinence criteria for its acceptance for sel-medication. Development of an information policy for medical products". Final Report, 2002.

*8. SÁNCHEZ, R.; RUPÉREZ, O.; DELGADO, M.A. y cols.: "Prevalencia de la incontinencia urinaria en la población mayor de 60 años atendida en atención primaria". Atención Primaria, 24: 241, 1999.

*9. WEIN, A.J.; ROVNER, E.: "Definition and epidemiology of overactive bladder". Urology, 60: 7, 2002.

10. ABRAMS, P.; FREEMAN, R.; ANDERSON, C. y cols.: "Mattiasson A. Tolterodine, a new antimuscarinic agent: as effective but better tolerated than oxibutynin in patients with an overactive bladder". BJU, 81: 801, 1998.

11. FULTZ, N.H.; HERZOG, R.: "Epidemiology of urinary symptoms in the geriatric population". Geriatr. Urolo., 23: 1, 1996.

12. HARRISON, G.L.; MEWMEL, D.S.: "Urinary incontinence in women: its prevalence and its management in a health promotion clinic". Br. J. Gen. Pract., 44: 149, 1994

13. MALMSTEN, U.G.; MILSOM, I.; MOLANDER, U. y cols.: "Urinary incontinence and lower urinary tract symptoms: an epidemiological study of men aged 4599 years". J. urol., 158: 1733, 1997.

14. INE. Instituto Nacional de Estadística. Censo Municipal de 2000. En: http://www.ine./

**15. STEWART, W.F.; VAN ROOYEN, J.B.; CUNDIFF, G.W. y cols.: "Prevalence and burden of overactive bladder in the United States". World J. Urol., 20: 327, 2003.

*16. CORCOS, J.; SCHICK, E.: "Prevalence of overactive bladder and incontinence in Canada". Can. J. Urol., 11: 2278, 2004. 